\title{
Risk factors for suicide in the elderly: What do we know? What do we need to find out?
}

\author{
By Margda Wærn
}

\section{This overview is based on a presentation with the same title given at a Danish conference on Suicide Prevention in Late Life, Aalborg 2009, with the inclusion of some updated references.}

\section{Introduction}

High rates of suicide are reported in elderly populations in most countries. According to pooled WHO data, the average global rate for males aged 75 years and above is 55.7 per 100000 , and the corresponding figure for females 18.8. (O'Connell et al., 2004). Considering the magnitude of these rates, it is remarkable that studies focusing specifically on suicide in late life were few, and primarily descriptive in nature until the year 2000.

The last decade has seen the advent of a new literature on suicide in later life. It should be pointed out, however, that age criteria vary from study to study. While some authors define "later life" as a period of life that starts at 50 , others set inclusion age at 60 or 65 .

Table 1 shows controlled psychological autopsy studies with a focus on later life published since 2000. These studies emanate from different parts of the world, including North America, Europe, Asia and Australia. Researchers have utilized data from interviews with close informants of suicide decedents and other sources in order to identify biological, psychological and social correlates of suicide in older people. These methodologically sound studies generate risk estimates, informing clinicians and public health planners about the magnitude of risk associated with various correlates of suicide. The use of qualitative study design is another important development in late-life psychological autopsy-based research. Kjölseth and colleagues (2009) carried out a psychological autopsy study based on qualitative interviews with informants, providing important insights into the development of the suicidal process in late life.

While the psychological autopsy approach has been shown to generate valid diagnoses (Conner et al., 2001a) and reliable information on life events, social support and suicidal behavior (Conner et al., 2001b), the approach does have its drawbacks (Hawton et al., 1998).

Results may be colored by the informants' grief reactions. Further, data collection is time-consuming and sample size is often a limitation. There are also concerns regarding control groups. The composition of comparison groups varies among studies. Results from studies utilizing a general population comparison group cannot be directly compared with those derived from studies that recruit comparison subjects from clinical samples/ natural deaths. Also, differential participation rates between cases and controls hamper interpretation.
Other research approaches are needed, especially when endeavoring to test hypotheses that require large study power. A number of recent studies based on national registers (Erlangsen et al., 2004, 2005, 2006, 2008), multicenter data (De Leo et al., 2001) and other large data bases (Juurlink et al., 2004), Kaplan et al., 2007, Voaklander et al., 2008) have generated risk data on suicide in late life, further enriching the literature. The aim of this overview is to take stock of what we have learned about risk factors for late life suicide during the past decade. What do we know? What do we need to find out?

\section{What do we know?}

\section{Mental disorders}

Depression is the only risk factor that was unanimously identified in all of the above cited case-controlled psychological autopsy studies. Among persons who commit suicide in later life, 65-75\% suffer from depression at the time of death (O'Connell et al., 2004). Risk estimates varied widely by setting, which in part relates to methodological inconsistencies, and to differences in base rates of depression in the comparison populations. Rates of depression in older persons who commit suicide are clearly higher than those seen in younger samples, and

Table 1. Controlled psychological autopsy studies with a focus on later life.

\begin{tabular}{l|l|l|}
\hline Authors & Setting & Year \\
\hline Conwell et al. & USA & 2000 \\
\hline Harwood et al. & UK & 2001 \\
\hline Beautrais & New Zealand & 2002 \\
\hline Wærn et al. & Sweden & 2002 \\
\hline Chiu et al. & Hong Kong & 2004 \\
\hline Duberstein et al. & USA & 2004 \\
\hline Préville et al. & Canada & 2005 \\
\hline
\end{tabular}

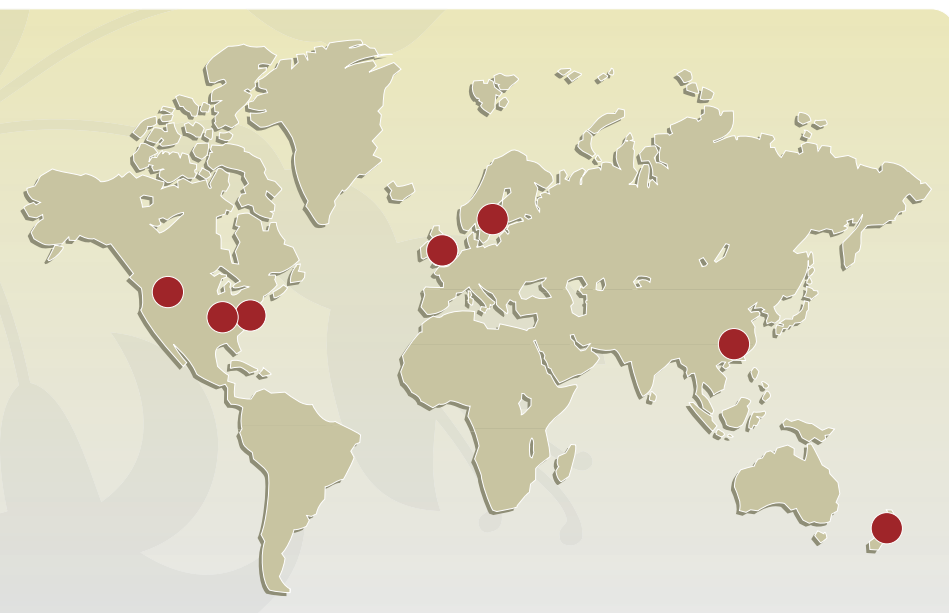




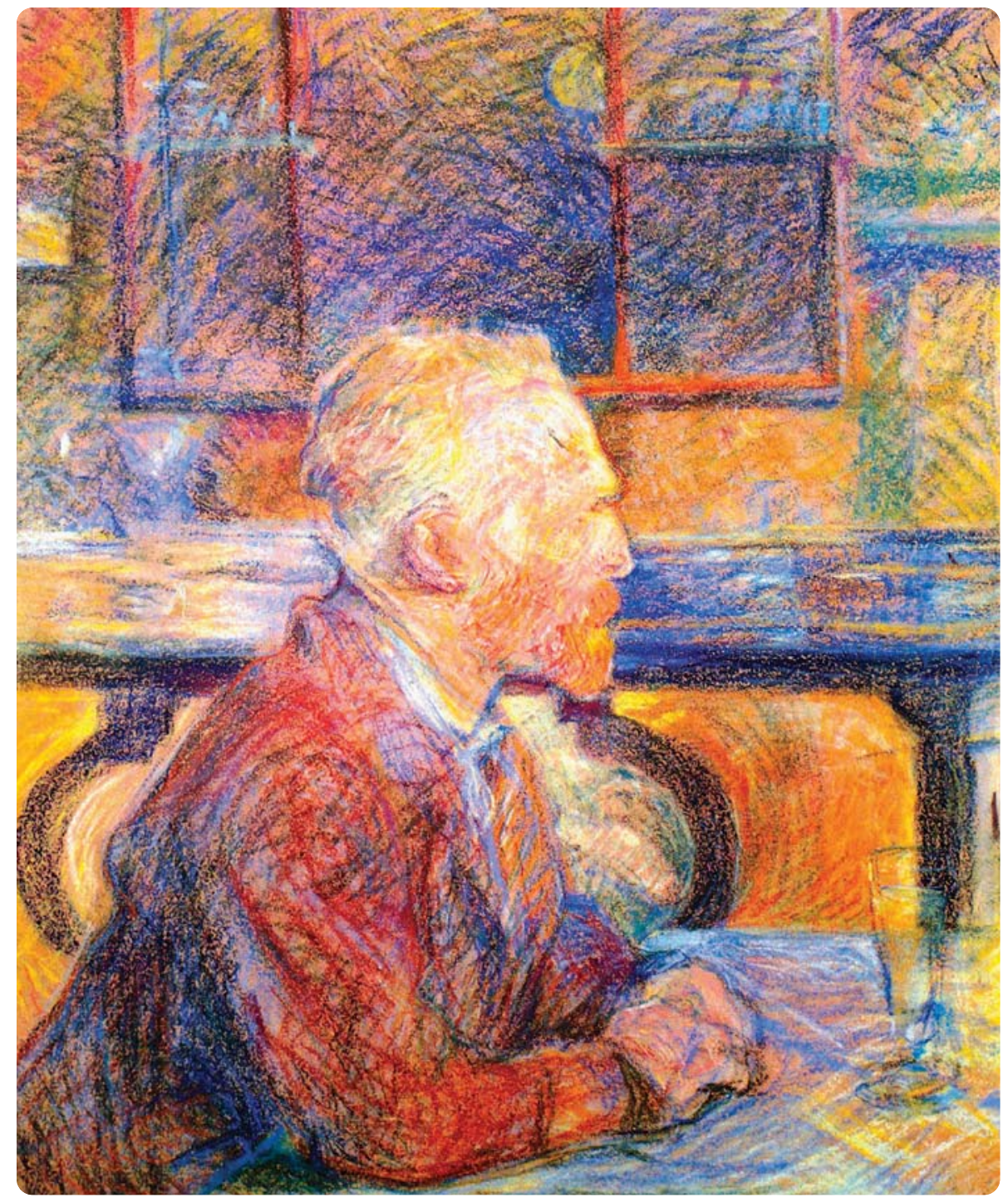

Portrait of Vincent Van Gogh (Henri de Toulouse-Lautrec, 1887)

the identification and treatment of depression is a major target for late-life suicide prevention. One study estimated that three quarters of elderly suicidal behaviour could be prevented were it possible to eradicate depression

(Beautrais et al., 2002) - in other words, a population attributable risk (PAR) for depression of $75 \%$. In comparison, the PAR for social factors was $27 \%$ in that study.

Regarding specific risks by depression subtype, the literature is sparser. One study that did examine subtypes (Waern et al., 2002a) found increased risk in both first episode and recurrent depression, the latter being more prominent than the former. In that same study,
$18 \%$ of the suicide cases fulfilled DSM IV criteria for a research diagnosis of minor depression. Thus, prevention programs need to target also elderly who do not fulfil criteria for clinical depression. Some elderly who die by suicide may have developed depressed mood in response to age-related existential situations. The majority of elderly in the qualitative study by Kjølseth and colleagues (2010a) were not perceived as depressed by the informants who knew them, although depressive symptoms were described in some cases.

Results are mixed regarding other types of mental disorders. In general, rates are lower than those cited above for depression. In the Swedish study, anxiety disorders were identified in $15 \%$ of the suicide cases and in $4 \%$ of the comparison subjects, yielding an odds ratio of 3.6 (Waern et al., 2002a). A tenfold increase in suicide risk was associated with psychotic disorder in that same study. Personality disorders were assessed in only one of the controlled late life psychological autopsy studies (Harwood et al., 2001). While uncommon, such disorders were more frequent among suicide cases than among persons in the comparison group, who died by natural death while in hospital.

Alcohol use disorders comprise the second most common diagnostic group in mixed-age suicides. Results from studies focusing specifically on suicides that occur in later life are mixed. In the studies set in Britain (Harwood et al., 2001) and in Hong Kong (Chiu et al., 2004), alcohol use disorders were uncommon in both cases and comparison subjects. While a somewhat higher proportion $(8 \%)$ was reported in the study from Canada (Preville et al., 2005), this proportion was similar to that observed in the control group (5\%). Studies from the US, (Conwell et al., 2000), New Zealand (Beautrais, 2002), and Sweden (Waern, 2003) demonstrated increased risk. Only the latter study examined risk in men and women separately. A significant association between alcohol use disorder and suicide remained in both sexes after adjustment for a number of potential confounders; an 8-fold risk increase was noted for men and a 13fold risk increase in women. These estimates of risk increase are not unlike the ten-fold increase reported from a metaanalysis on alcohol use disorders in mixed-age cohorts (Wilcox et al., 2004). Turvey and colleagues utilized data from a large prospective community-based study of over 14000 individuals aged 65 (Turvey et al, 2002). No associations could be shown between suicide and 1) frequency and 2) quantity of alcohol consumption in this cohort.

The retrospective diagnosis of dementia constitutes a particular challenge in psychological autopsy-based research. 
Only one of the above-cited controlled psychological autopsy studies (Harwood et al., 2001) included a structured instrument to measure cognitive impairment by proxy. Dementia was observed in four of the 100 cases, and other forms of organic disorders were reported in seven. Rates were significantly higher in the comparison group, which was comprised of hospitalized persons who died from natural causes. A second study involving deceased control subjects (Preville et al., 2005) did not show rates of dementia, but confusion at the time of death was reported in $11 \%$ of the suicides and $8 \%$ of the controls. The psychological autopsy studies that have utilized living control subjects drawn from the general population reported similar rates of dementia in cases and comparison subjects (Haw et al., 2009). One register study based on a total national cohort of persons aged 50 and above demonstrated increased risk when a diagnosis of dementia was made during hospitalisation, especially in younger patients (Erlangsen et al., 2008). However, the risk associated with dementia was significantly lower than that associated with other mental disorders (Erlangsen et al., 2006). Focusing on cognitive function in a clinical cohort, Dombrovski and colleagues (2008) assessed cognition in suicidal depressed and non-suicidal depressed persons aged 60 and above and found that poor performance on tests of executive function, attention and memory were associated with suicidal behaviour. As cognitive function is particularly difficult to assess by proxy, studies such as this one can help to clarify associations. Another approach is to study neuroanatomical changes related to neurodegenerative disease. Increased levels of changes related to Alzheimer's disease were reported from a small study that compared pathology in suicides and natural deaths (Rubio et al., 2001). This could not be corroborated in a larger study in which the control group was comprised of persons who died accidental deaths, however (Pesiah et al., 2007).

Summarizing the recent literature on mental disorders in late life suicide, depression has been identified as the strongest diagnostic correlate of suicide in later life. In some settings, alcohol use disorders are also associated with strong risks. Most of those who commit suicide in later life suffer from some type of mental disorder ( $\mathrm{O}^{\prime}$ Connell et al., 2004). For older adults $(60+)$ who were hospitalized with psychiatric disorders, over half of the suicide deaths took place within a week of admission or discharge, identifying these time periods as particularly risky (Erlangsen et al., 2006).

\section{Physical illness and disability}

Physical illness was shown to be associated with suicide in several of the controlled studies (Conwell et al. 2002, Waern et al., 2002b). In the latter study, neurological disorders, cancer and vision problems were specific disorders that were associated with increased suicide risk. Further, the association between physical illness and suicide was independent of depression. In connection with a large register-based study that used specific types of medication as a proxy indicator of various illnesses, Juurlink and colleagues (2004) reported increased suicide risk in congestive heart disease, chronic lung disease, and seizure disorders. Somewhat surprisingly, Parkinson's disease was not associated with increased risk in that study, despite high rates of depression in afflicted individuals. Stroke, cancer and liver disease were the disorders shown to associate with suicide in another large register study (Voaklander et al 2008). Another question is whether it is physical illness per se or rather the resulting functional limitations that impact on suicide risk. The authors of a large study based on a nationally representative sample (Kaplan et al., 2007) reported that physical illness was no longer a risk factor for suicide, when functional limitation was included in the model. Impairment in activities of daily living is associated with suicide, and the relationship is independent of depression (Conwell et al., 2010). Elders who committed suicide in that study were more likely to be in contact with home care nurses and home help providers.
The latter was the case for the 75+ cohort in the Swedish psychological autopsy study, as well (Waern et al., 2003.) In a qualitative psychological autopsy study, informants who had known the deceased persons well described how functional decline resulted in a loss of freedom of action and self-determination (Kjølseth et al., 2010), indicating that these types of experiences may constitute important determinants of suicide in later life.

\section{Social factors}

Living alone was associated with a fivefold increase in suicide risk in one of the psychological autopsy studies (Conwell et al., 2000). Such a relationship could not be shown in the other studies. Spousal bereavement predicted suicide in older men, but not in women (Erlangsen et al., 2004). The risk associated with bereavement was particularly high in men aged 80 and above, and risk remained elevated years after the loss of the spouse.

In the British psychological autopsy study, (Harwood et al., 2001), interpersonal problems contributed to the suicide in almost a third of the cases. Family discord predicted suicide risk (Rubenowitz et al., 2001), and this association was independent of depression diagnosis. Interpersonal conflict was shown to be significantly more common in older persons who commited suicide than in the background population (Beautrais, 2002, Duberstein et al., 2004). Conwell and colleagues (2000) reported lower social interaction in primary care attendees who committed suicide than in those who did not. In the New Zealand study, low social interaction predicted suicide, and the association remained even after both mental and physical health were taken into consideration (Beautrais, 2002). In the qualitative psychological autopsy study, decedents were described as persons who kept a distance in their relationships, both in family relationships as well as in other social contexts (Kjølseth et al., 2009). Antonovsky's concept of Sense of Coherence (SOC) could be of relevance in this connection. Among persons aged 
70 and above who were interviewed in connection with care after a suicide attempt, low social interaction was associated with low Sense of Coherence (Mellqvist et al., 2010). SOC has been shown to be related to suicidal behaviour in younger persons (Mehlum et al., 1998). As yet, it remains unclear whether such an association is the case among elderly. One psychological autopsy study reported social factors associated with decreased suicide risk (Rubenowitz et al., 2001). Active club membership and pursuing a hobby appeared to be protective factors.

\section{Personality}

Only one of the controlled psychological autopsy studies shown in Table 1 provided risk data regarding personality features in suicide cases and controls. The British study (Harwood et al., 2001) reported that obsessive-compulsive and anxious traits were more common among suicide cases than those who died natural deaths in hospital. Traits described by next-of-kin in the qualitative psychological autopsy study of elderly Norwegian males who committed suicide included a need to be in control, and a tendency to link self-esteem with activity and achievement (Kjølseth et al., 2009). Informants in that study reported that the elderly men were emotionally closed, and kept a distance.

In an older, uncontrolled study, low openness to experience and rigidly defined self concept characterized older persons who died by suicide (Duberstein et al. 1994). More recently, prospective clinical studies focusing on older suicide attempters demonstrated that those who later died by suicide had scored lower in neuroticism, and were more conscientious (Tsoh et al., 2005, Useda et al., 2007) than those who did not take their lives. Further, they were less open to new experiences and more agreeable (Tsoh et al. 2005), more achievement oriented, and less openness to fantasy and aesthetics (Useda et al., 2007).

\section{Previous suicidal behavior}

While older persons are more likely to commit suicide than younger, they are

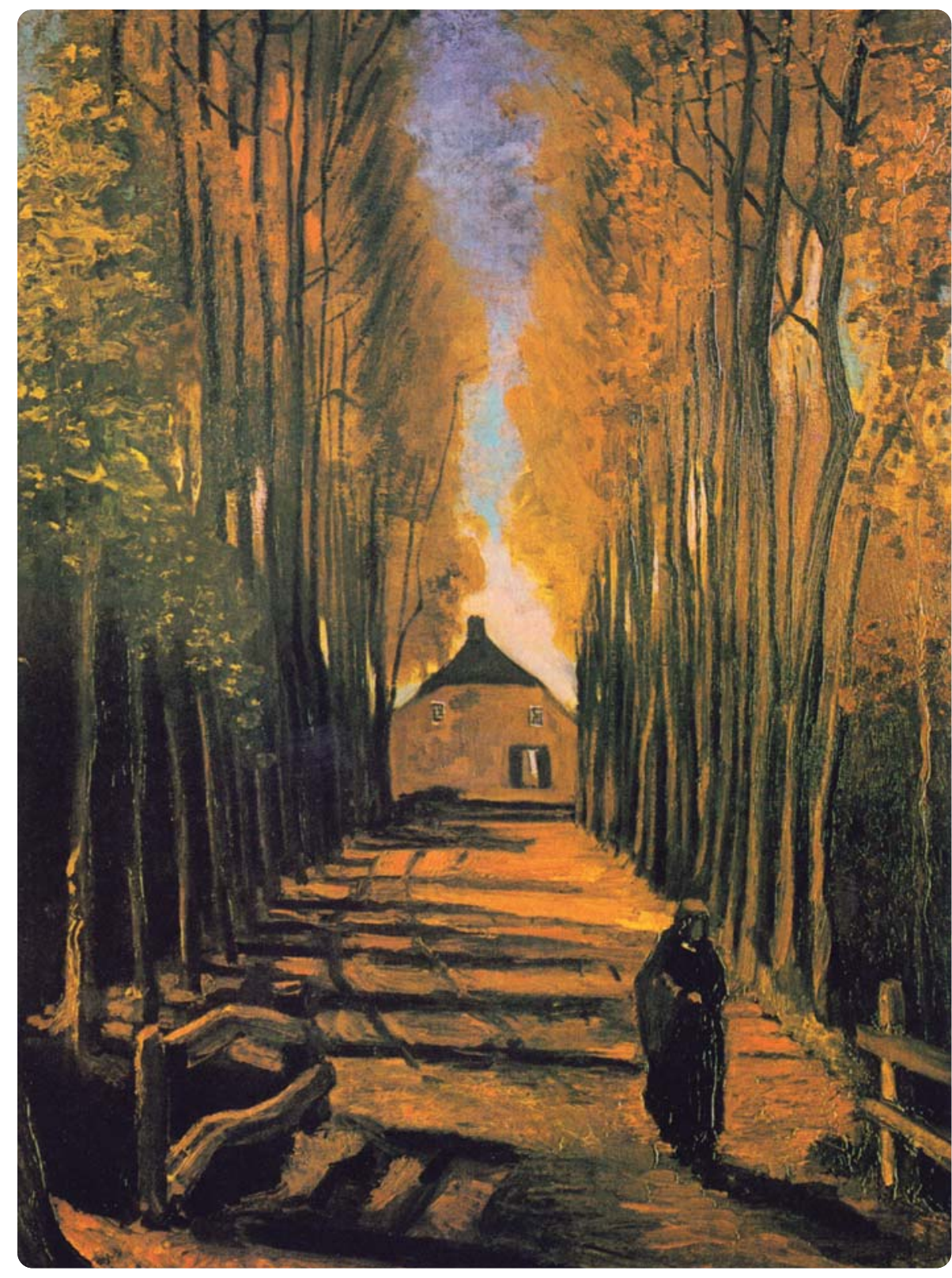

Avenue of Poplars in Autumn (Vincent van Gogh, 1864)

less likely to attempt suicide. The rateratio of attempted to completed suicide was shown to decrease from more than 100 in teenagers to less than 10 in those aged 60 and above (Hawton and Harris, 2008). Regarding findings from the recent late-life psychological autopsy studies, proportions with previous attempts varied markedly among settings (from $2-3 \%$ to $42 \%$ ). Rates of attempted suicide among the comparison groups employed in those studies varied as well, and odds ratios ranged from 40 (Chiu et al., 2004) to 186 (Voaklander et al.,
2008). In a prospective investigation of older deliberate self-harm patients, self-harm prior to the index episode was the main risk factor for subsequent death by suicide (Hawton and Hariss, 2006).

\section{Prescription drugs and suicide in late life}

While concerns regarding the possible induction of suicidal behaviour by SSRI antidepressants resulted in a "black box warning" for young people, these drugs have been associated with reduced risk of suicide in elderly people (Barak et al., 
2006, Barbui et al., 2008). Focusing also on other types of psychoactive drugs, a large Canadian cohort study showed that benzodiazepines were associated with increased suicide risk (Voaklander et al., 2008). The authors pointed out that this could be due to confounding by indication. Using detailed diagnostic information from their psychological autopsy study on suicides in persons aged 65 and above, Carlsten and Waern (2009) showed that both sedatives and hypnotics were associated with suicide risk, also after adjustment for confounding by psychiatric disorders.

\section{Availability of suicide methods}

A partial explanation for the increased risk associated with sedatives and hypnotics noted above might be that the drugs were readily available and could be utilized in connection with a lethal overdose. Reduced availability is an evidence-based suicide preventive strategy. In the US, where most suicides involve shooting, Conwell and colleagues (2002) demonstrated that having a gun in the home was associated with a twofold increase in suicide risk in older adults, suggesting that restrictive gun policies could reduce risk.

\section{What do we need to find more about?}

The above overview demonstrates that late life suicide research has now moved well beyond the descriptive case series of the twentieth century. However, challenges remain. In particular, we need to find out more about factors associated with suicide in the very elderly, and how the suicidal process develops over time in this age group. In this connection, qualitative studies will make an essential contribution, by providing a different type of information than that produced in connection with traditional risk factor research.

Subthreshold disorders are common in elderly populations, and particular symptom clusters may be associated with suicide risk even in the absence of a fullblown syndrome. Symptoms of interest may include anxiety, anhedonia, rumination, sleep-related problems and cognitive dysfunction. As pointed out by Haw and colleagues in their recent review (2009), there is a lack of studies focusing specifically on possible associations between suicide and specific types of dementia including vascular dementia, frontotemporal, Lewy body and HIV dementia.

We need to find out more about how the above-reviewed biological, psychological and social factors interact to further accentuate risk. The role of personality traits is pertinent in this connection, and larger studies are required to elucidate interactions among personality traits, social factors and biological and behavioral markers of vulnerability. The latter include serotonergic neurotransmission and trait aggression/ impulsivity. Explorative data from the Swedish psychological autopsy study (Waern 2005) points to a familial vulnerability. In $15 \%$ of the cases, suicide had occurred in some other first degree family member prior to the elderly person's death by suicide. Previous episodes of suicidal behavior were more common among those elderly persons who lost a family member by suicide than in those who did not, suggesting a heritable vulnerability, a suicidal diathesis. Increased understanding of the neurobiology and genetics of late-life suicide can help to elucidate etiology, and in time lead to the development of more individualized pharmaceutical treatments. Further, it has been shown that depressed inpatients aged 65 years and above who do not suppress cortisol are more likely to commit suicide than those that do (Jokinen and Nordström 2008). Multicenter studies would be needed to determine the clinical usefulness of the dexamethasone suppression test as a complementary tool in suicide risk assessment in the elderly.

While prevention is not a primary focus of this review, the above-cited studies demonstrate that psychiatric disorders in general and depression in specific are important targets for late life suicide prevention. However, there is a large degree of heterogeneity among elders who attempt and commit suicide, and it is clear that multifaceted prevention strategies are needed, as is the case in suicide prevention in general (Hawton \& van Heeringen, 2009). Large multicenter studies will be required to test intervention outcomes, and there will be a need for the development of consensus protocols regarding inclusion criteria, diagnostic tools and structured instruments to determine outcomes including consensus definitions of suicide attempts and completed suicide.

At the same time, qualitative studies are needed to provide new knowledge that large multicenter studies cannot generate. What are the driving forces of hopelessness and suicidality in the perspective of the elderly person? How can the health care system and other service providers better meet their perceived needs (Kjölseth et al., 2010)? What kinds of interventions might help to ameliorate existentially-related reactions to the burdens of old age, making life more worth living?

In concluding, a number of correlates of late-life suicide have been identified through risk factor research during the latest decennium. Findings can inform clinicians and others who provide services for the elderly. Further, results from these studies contribute to the scientific basis for large intervention studies. At the same time, smaller, more focused studies are needed, including those that can tap on to the existential aspects of suicidality in the elderly. In the words of the artist Edvard Munch: "It is not we who die, it is the world that dies away from us." Prevention strategies tailored specifically to suit the elderly may widen the world by strengthening reasons for living in old age.

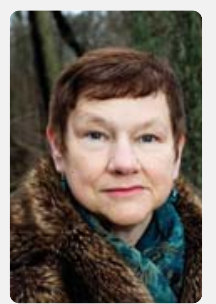

Margda Wærn, MD, PhD is professor of psychiatry with a special focus on suicidology at the Section for Psychiatry and Neurochemistry, University of Gothenburg. She is also a senior consultant in psychiatry at Sahlgrenska University Hospital. 


\section{References}

Barak Y, Aizenberg D. (2006). Association between antidepressant prescribing and suicide in Israel. International Clinical Psychopharmacology, 21, 281-284.

Barbui C, Esposito E, Cipriani A. (2009). Selective serotonin reuptake inhibitors and risk of suicide: a systematic review of observational studies. Canadian Medical Association Journal,180, 291-297.

Beautrais AL. (2002). A case control study of suicide and attempted suicide in older adults. Suicide and Life-Threatening Behavior, 32, 1-9.

Carlsten A, Waern M. (2009). Are sedatives and hypnotics associated with increased suicide risk of suicide in the elderly? BMC Geriatrics, 9, 20.

Chiu HFK, Yip PS, Chi I, Chan S, Tsoh J, Kwan CW, Li SF, Conwell Y, Caine E. (2004). Elderly suicide in Hong Kong: a case-controlled psychological autopsy study. Acta Psychiatrica Scandinavica, 109 299-305.

Conner K, Conwell Y, Duberstein PR. (2001a). The validity of proxy-based data in suicide research: A study of patients 50 years of age and older who attempted suicide. II. Life events, social support and suicidal behavior. Acta Psychiatrica Scandinavica, 104, 452-457.

Conner K, Duberstein P, Conwell Y. (2001b). The validity of proxy-based data in suicide research: A study of patients 50 years of age and older who attempted suicide. I. Psychiatric diagnoses. Acta Psychiatrica Scandinavica, 104, 204-209.

Conwell Y, Lyness JM, Duberstein P, Cox C, Seidlitz L, DiGiorgio A, Caine ED. (2000). Completed suicide among older patients in primary care practices: a controlled study. Journal of the American Geriatrics Society, 48, 23-29.

Conwell Y, Duberstein PR, Caine ED. (2002). Risk factors for suicide in later life. Biological Psychiatry, $52,193-204$

Conwell Y, Duberstein PR, Hirsch JK, Conner KR, Eberly S, Caine ED. (2010). Health status and suicide in the second half of life. International Journal of Geriatric Psychiatry, 25, 371-379.

De Leo D, Padoani W, Scocco P, Lie D et al. (2001). Attempted and completed suicide in older subjects: results from the WHO/EURO Multicentre study of suicidal behaviour. International Journal of Geriatric Psychiatry, 16, 300-310.

Dombrovski AY, Butters MA, Reynolds CF III Houck PR, Clark L, Mazumdar S, Szanto K. (2008). Cognitive performance in suicidal depressed elderly: Preliminary report. American Journal Geriatric Psychiatry, 16, 109-115.

Duberstein PR, Conwell Y, Caine ED. (1994). Age differences in the personality characteristics of suicide completers: Preliminary findings from a psychological autopsy study. Psychiatry, 57, 213-224.

Duberstein PR, Conwell Y, Conner KR, Eberly S, Caine ED. (2004). Suicide at 50 years of age and older: perceived physical illness, family discord and financial strain. Psychological Medicine, 34, 137-146.

Erlangsen A, Jeune B, Bille-Brahe U, Vaupel JW. (2004). Loss of partner and suicide risks among oldest old: a population-based register study. Age and Ageing, 33, 378-383.
Erlangsen A, Vach W, Jeune B. (2005). The effect of hospitalization with medical illnesses on the suicide risk in the oldest old: a population-based register study. J Am Geriatr Soc, 53, 771-776.

Erlangsen A, Zarit SH, Tu X, Conwell Y. (2006). Suicide among older psychiatric inpatients: an evidence-based study of a high-risk group. American Journal of Geriatric Psychiatry, 14, 734-741.

Erlangsen A, Zarit SH, Conwell Y. (2008). Hospitaldiagnosed dementia and suicide: a longitudinal study using prospective, nationwide register data. American Journal of Geriatric Psychiatry, 16 220-228.

Harwood D, Hawton K, Hope T, Jacoby R. (2001). Psychiatric disorders and personality factors associated with suicide in older people: a descriptive and case-control study. International Journal of Geriatric Psychiatry, 16, 155-165.

Harwood DM, Hawton K, Hope T, Harriss L, Jacoby R. (2006). Life problems and physical illness as risk factors for suicide in older people: a descriptive and case-control study. Psychological Medicine, $36,1265-1274$.

Haw C, Harwood D, Hawton K. (2009). Dementia and suicidal behavior: a review of the literature. International Psychogeriatrics, 21, 440-453.

Hawton K et al. (1998). The psychological autopsy approach to studying suicide: a review of methodological issues. Journal of Affective Disorders, 50, 269-276.

Hawton K, Hariss L. (2006). Deliberate self-harm in people aged 60 years and over: characteristics and outcome of a 20-year cohort. International Journal of Geriatric Psychiatry, 21, 572-581.

Hawton K, Hariss L. (2008). How does deliberate self-harm occur relative to each suicide? A study of variations by gender and age. Suicide and lifethreatening behavior, 38, 650-660.

Hawton K, van Heeringen K. (2009). Suicide. Lancet, 373, 1372-1381.

Jokinen J, Nordström P. (2008). HPA axis hyperactivity as suicide predictor in elderly mood disorder patients. Psychoneuroendocrinology, 33, 1387-1393.

Juurlink DN, Herrmann N, Szalai JP, Kopp A,

Redelmeier DA. (2004). Medical illness and the risk of suicide in the elderly. Archives of Internal Medicine, 164, 1179-1184.

Kaplan MS, McFarland BH, Huguet N, Newson. (2007). Physical illness, functional limitations, and suicide risk: a population-based study. American Journal of Orthopsychiatry, 77, 56-60.

Kjølseth I, Ekeberg Ø, Steihaug S. (2009). "Why do they become vulnerable when faced with the challenges of old age?" Elderly people who committed suicide, described by those who knew them. International Psychogeriatrics, 21, 903-912. Kiølseth I, Ekeberg Ø, Steihaug S. (2010). Why suicide? Elderly people who committed suicide and their experience of life in the period before death. International Psychogeriatrics, 22, 209-218.

Kjølseth I, Ekeberg $\varnothing$, Steihaug S. (2010). Elderly people who committed suicide - their contact with the health service. What did they expect, and what did they get? Aging and mental health, 14 , 938-946.
Mellqvist M, Wiktorsson S, Skoog I, Östling S, Waern M. (2010). Sense of coherence in elderly suicide attempters - the impact of social and health related factors. Int Psychogeriatrics, Mar 1:1-8.

Mehlum L. (1998). Suicidal ideation and sense of coherence in male conscripts. Acta Psychiatrica Scandinavica, 98, 487-492.

O'Connell H, Chin AV, Cunningham C, Lawlor B. (2004). Recent developments: suicide in older people. British Journal of Medicine 329, 895-899. Peisah C, Snowdon J, Gorrie C, Kril J, Rodriguez M. (2007). Investigation of Alzheimer's diseaserelated pathology in community dwelling older subjects who committed suicide. Journal of Affective Disorders, 99, 127-132.

Préville M, Hébert R, Boyer R, Bravo G, Seguin M. (2005). Physical health and mental disorder in elderly suicide: a case-control study. Aging \& Mental Health, 9, 576-584.

Rubenowitz E, Waern M, Wilhelmson K, Allebeck P. (2001). Recent life events and suicide in the elderly. Psychological Medicine, 31,1193-1202.

Rubio A, Vestner AL, Stewart JM, Forbes NT, Conwell Y, Cox C. (2001). Suicide and Alzheimer's pathology in the elderly: a case-control study. Biological Psychiatry, 49, 137-145.

Tsoh J, Chiu HF, Duberstein PR, Chan SS, Chi I, Yip PS, Conwell Y. (2005). Attempted suicide in elderly Chinese persons: a multi-group, controlled study. American Journal of Geriatric Psychiatry, 13, 562-571.

Turvey CL et al. (2002). Risk factors for late-life suicide: prospective, community-based study. American Journal of Geriatric Psychiatry, 10, 398-406.

Useda JD, Duberstein PR, Conner KR, Beckman A, Franus N, Tu X, Conwell Y. (2007). Personality differences in attempted suicide versus suicide in adults 50 years of age and older. J Consult Clin Psychol 75, 126-133.

Voaklander DC, Rowe BH, Dryden DM, Pahal J, Saar P, Kelly KD. (2008). Medical illness, medication use and suicide in seniors: a population-based case-control study. Journal of Epidemiological Community Health, 62, 138-146.

Waern M, Runeson B, Allebeck P, Beskow J, Rubenowitz E, Skoog I, Wilhelmson K. (2002a). Mental illness in elderly suicides: A controlled study. American Journal of Psychiatry, 159, 450-455.

Waern M, Rubenowitz E, Runeson B, Skoog I, Wilhelmson K, Allebeck P. (2002b). Illness burden in elderly suicides: A controlled study. British Medical Journal, 324, 1355-1358.

Waern M. (2003). Alcohol dependence and abuse in elderly suicides. Alcohol and alcoholism, 38, 249-254.

Waern M, Rubenowitz Lundin E, Wilhelmson K. (2003). Risk factors for suicide over 75 . Gerontology, 49, 328-334.

Waern M. (2005). Suicide among family members of elders who commit suicide: An exploratory study. Suicide and Life-Threatening Behavior, 35, 356-364.

Wilcox HC, Conner KR, Caine ED. (2004).

Association of alcohol and drug use disorders and completed suicide: an empirical review of cohort studies. Drug Alcohol Dependence, 7, Suppl, S11-19. 